\title{
Are the effects of IVF on DNA methylation driven by intracytoplasmic sperm injection and male infertility?
}

\author{
"...evidence tells us that both genetic and epigenetic defects could be \\ inherited from infertile men via intracytoplasmic sperm injection."
}

First draft submitted: 11 April 2016; Accepted for publication: 14 April 2016; Published online: 1 July 2016

Keywords: DNA methylation • epigenetic • fertility • in vitro fertilization

The term 'in vitro fertilization' (IVF) covers a number of related techniques in which an oocyte is joined with a sperm in vitro to fuse and form a zygote, which is later transferred to the woman's uterus, where it develops as an embryo. Here we briefly review what is known about the developmental and epigenetic consequences of IVF, then we focus on the specific question of whether the effects of IVF on DNA methylation could be driven by procedure of intracytoplasmic sperm injection (ICSI), used in a subset of IVFs.

IVF procedures have been around for almost 40 years and are widespread throughout the developed world, contributing to between 0.5 and $5 \%$ of live births [1]. Most IVF protocols involve ovarian stimulation, sperm and oocyte retrieval and preparation (including sperm washing), fertilization via coincubation, embryo culture and embryo selection and transfer [2]. There are many variations in IVF methods including cryopreservation of the embryo prior to transfer, assisted hatching and extended culture prior to transfer. In ICSI, sperm injection replaces coincubation.

In general, IVF has been linked with a slightly higher risk of birth defects such as spina bifida, cerebral palsy and abnormalities of the cardiovascular and musculoskeletal systems [3]. Some have found that such effects disappeared after controlling for other IVFassociated factors [4]. Others have linked IVF with a larger placental/birth weight ratio and higher levels of childhood cardiometabolic risk factors such as blood pressure and fasting glucose [5]. A recent meta-analysis of four studies found an almost fourfold incidence in imprinting disorders such as Angelman syndrome, Beckwith-Wiedemann syndrome and Prader-Willi syndrome, in IVF-conceived versus naturally conceived children [6].

Many animal and human studies have attempted to separate the effects of specific stages of IVF [7]. Culture media components such as oxygen concentration and amino acid composition affect embryo viability and although many studies have linked some culture media to low birth weight and adverse neurodevelopmental outcomes, others have not. One recent study of 73 IVFs randomized to different culture media showed that the choice of media can have an effect on neurodevelopmental outcomes in childhood [8]. In addition, extended culture prior to embryo transfer has been associated with an increased incidence of preterm birth but cryopreservation of embryos prior to transfer seems to avoid such risks.

We next consider whether IVF procedures interfere with epigenetic remodeling in early development. Between fertilization and implantation, most gamete-specific epigenetic marks, including DNA methylation, are removed [9]. Following implantation, levels of DNA methylation rise as tissue-specific epigenetic marks are established. In imprinted genes, expressed in somatic cells from a single parental allele, things are a little different. Epigenetic state at imprinting control
Epigenomics
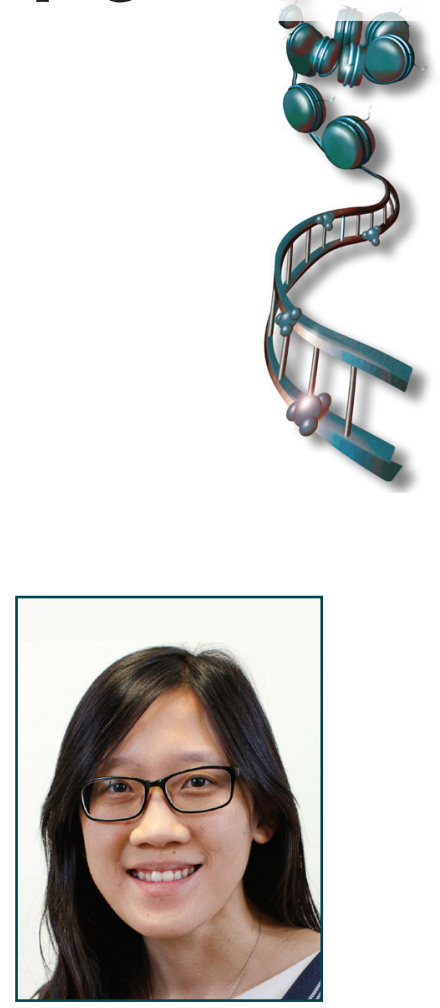

Yuk Jing Loke Murdoch Childrens Research Institute \& Department of Paediatrics, University of Melbourne, Royal Children's Hospital, Flemington Road, Parkville, Victoria 3052,

Australia

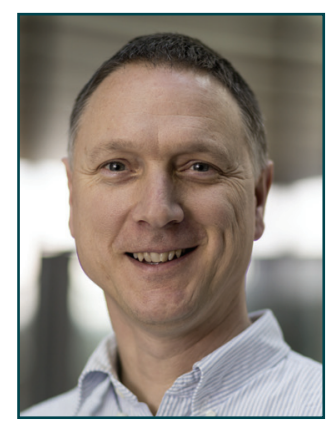

Jeffrey M Craig

Author for correspondence: Murdoch Childrens Research Institute \& Department of Paediatrics, University of Melbourne, Royal Children's Hospital, Flemington Road, Parkville, Victoria 3052, Australia

jeff.craig@mcri.edu.au

Future : Medicine ${ }_{\text {part of }}$ 
regions (ICRs) established in the germline are largely unaffected by postfertilization epigenetic remodeling. However, differentially methylated regions are established within imprinted loci in post-implantation somatic tissues from which time they contribute transcription within each imprinted locus.

Studies of the postfertilization effects of IVF procedures on DNA methylation have been reviewed recently $[6,10]$. Although common themes of disrupted methylation and/or expression of imprinted genes (e.g., IGF2, H19, MEST and KCNQ10T1) and disrupted global methylation have emerged, there has been no consensus on the direction, size and tissue specificity of effects. However, evidence has been presented that specific IVF procedures such as ovarian stimulation, retrieval of oocytes, sperm preparation and duration of embryo culture can influence epigenetic state of the embryo. Ovarian stimulation has also shown to affect the epigenetic state of the endometrium.

Recent attention has turned to ICSI as a source of developmental and epigenetic disruption. ICSI was developed to enable infertile males to contribute toward conception [11]. Infertile men can have too few sperm (oligospermia) or a lack of motile sperm (azoospermia). In the former, sperm can be isolated from semen. If the latter is nonobstructive, such as in defects of spermatogenesis, sperm can be surgically extracted from the testes. If obstructive, such as in vasectomized men, viable sperm can be aspirated from the epididymis.

\section{"Epigenetic defects in sperm from infertile men have included failure to fully remove maternal methylation imprints, failure to fully establish paternal methylation imprints, variation in the protamine levels and increased histone retention in sperm."}

Since it was introduced in 1992, the prevalence of ICSI has increased from around one-third of IVF treatments to around two-thirds or higher [11], reflecting a wider use outside male infertility. This increase has been due to many factors including the high rate of success of ICSI in relation to non-ICSI IVF but is not without controversy, which is centered around risks for adverse developmental outcomes [11]. A study of 3372 offspring conceived by ICSI (mainly due to male infertility) and 9016 offspring conceived naturally found a relative risk for congenital malformation of 1.44 (95\% CI: 1.25-1.6) for ICSI [12]. A study of 211 offspring conceived by ICSI and 195 conceived naturally found that the former had differences in finger length usually associated with later infertility [13]. A study of 116 male and 101 female adolescents and 223 naturally conceived controls found that breast development at the age of 14 years was less advanced in ICSI-conceived compared with naturally conceived girls [14]. A study of similar scale found that ICSI-conceived girls were more likely to be obese than those conceived naturally [15]. A significant association of ICSI with birth defects did not diminish after controlling for multiple factors [4], implicating zona puncture and/or male infertility as strong candidates for a cause of these effects. Other studies (e.g., [16]) have drawn conclusions about increased incidence of imprinting disorders associated with IVF/ICSI but have not differentiated between the effects of ICSI and non-ICSI IVF.

Few human studies have investigated the impact of ICSI on epigenetics. We compared DNA methylation in buccal cell DNA from 174 naturally conceived twin pairs with 20 ICSI-conceived twin pairs and 14 non-ICSI IVF twin pairs [17]. We found that differences in DNA methylation at the H19/IGF2 ICR between ICSI and naturally conceived newborns $(-3.8 \% ; \mathrm{p}=0.026)$ was driving the difference in DNA methylation between all IVF-conceived and naturally conceived newborns $(-3.5 \% ; \mathrm{p}=0.048)$. We found no evidence for difference in methylation between newborns conceived through non-ICSI IVF and natural means $(p=0.634)$ [17]. In a study comparing of X-inactivation in newborn placentas from 60 ICSI-conceived, 72 non-ICSI-conceived and 52 naturally conceived females, no evidence was found for a difference in DNA methylation patterns between the three groups [18].

Studies of rodents have begun to isolate the effects of ICSI procedures, fertility and genetics [19]. Three studies of congenic mouse strains comparing naturally conceived and ICSI-conceived, minimally cultured embryos found major differences in gene expression in tissues from embryos and/or newborns. One study found no differences in gene expression between naturally conceived newborns and 1 day of ex vivo embryo culture. Another observed a delay in the active demethylation of the male pronucleus in ICSI-conceived zygotes. Although these studies did not use non-ICSI IVF controls, they showed that some facet of the ICSI procedure other than fertility and embryo culture was influencing gene expression. This could be sperm handling/washing or zona puncture. No study has yet isolated the effects of these factors on epigenetics of the embryo or newborn. However, it is intriguing that multiple animal studies have found epigenetic defects in the male but not the female pronucleus after ICSI, implicating paternal origins of epigenetic dysfunction (reviewed in [19]).

Many have asked whether epigenetic differences in IVF-conceived offspring could be inherited transgenerationally from sperm. Entry of primordial germ cells into the gonad during gastrulation is accompanied by demethylation on a genomic scale, including imprinting 
ICRs. This is followed by a progressive increase in global methylation including at maternally imprinted genes such as PLAG1 in oocytes and paternally imprinted loci such as IGF2/H19 in sperm (reviewed in [20]). At the same time, histone $\mathrm{H} 3$ and $\mathrm{H} 4$ deacetylation occurs at a global level. From the onset of puberty, spermatogenesis via meiotic divisions is accompanied by an increase in histone $\mathrm{H} 3$ and $\mathrm{H} 4$ acetylation, incorporation of histone variants and replacement of a most but not all histones by protamines.

Male infertility, most commonly manifested as nonobstructive azoospermia, has been associated with epigenetic defects at each of the above stages of spermatogenesis (reviewed in [20-22]). Epigenetic defects in sperm from infertile men have included failure to fully remove maternal methylation imprints, failure to fully establish paternal methylation imprints, variation in the protamine levels and increased histone retention in sperm. One study identified genome-wide hypermethylation in sperm including at nonimprinted genes associated with embryonic development and at tandem repeats [23]. Recently, sperm prepared by different methods were found to differ epigenetically [24]. Density gradient preparation, frequently used for IVF, was associated in sperm with decreased DNA methylation in transcription factor genes and decreased histone retention in genes associated with neural development, compared with untreated sperm. Genetic defects detected in the sperm of infertile men include deletion of heterochromatin, and an increase in DNA fragmentation, telomere length and apoptosis [25]. Taken together, this evidence tells us that both genetic and epigenetic defects could be inherited from infertile men via ICSI. In evidence, some studies have found similar epigenetic defects in infertile men and their offspring. Such data have come from case reports and focused on specific imprinted loci $[26,27]$.

\section{References}

1 Pinborg A, Wennerholm UB, Romundstad LB et al. Why do singletons conceived after assisted reproduction technology have adverse perinatal outcome? Systematic review and meta-analysis. Hum. Reprod. Update 19(2), 87-104 (2013).

2 Balaban B, Sakkas D, Gardner DK. Laboratory procedures for human in vitro fertilization. Semin. Reprod. Med. 32(4), 272-282 (2014).

3 Hansen M, Kurinczuk JJ, Milne E, De Klerk N, Bower C. Assisted reproductive technology and birth defects: a systematic review and meta-analysis. Hum. Reprod. Update 19(4), 330-353 (2013).

4 Davies MJ, Moore VM, Willson KJ et al. Reproductive technologies and the risk of birth defects. N. Engl. J. Med. 366(19), 1803-1813 (2012).
In summary, despite the links between male infertility and zona puncture and abnormal DNA methylation and limited evidence that ICSI may result in more severe epigenetic defects on offspring than non-ICSI IVF, we are far from conclusively answering our original question of whether the effects of IVF on DNA methylation are driven by ICSI or male infertility. To accomplish this, we call for more longitudinal cohorts, ideally in which subjects are recruited preconception at which time specific details of male and female infertility and specific details of IVF treatments are carefully recorded. We also call for epigenome-scale analysis of DNA methylation, histone modification and gene expression from sperm, embryo and from multiple tissues of the offspring. In addition, we need more animal models that have the ability to separate the each component of IVF, for example those that can separate the effects of male infertility zona puncture used in ICSI. It is imperative that we gather this data because of the everwidening use of ICSI for IVF.

\section{Acknowledgements}

The authors thank J Halliday for helpful discussions on this manuscript.

\section{Financial \& competing interests disclosure}

JM Craig is supported by the Australian National Health and Medical Research Council and the Financial Markets Foundation for Children. The MCRI is supported by the Victorian Government's Operational Infrastructure Support Program. The authors have no other relevant affiliations or financial involvement with any organization or entity with a financial interest in or financial conflict with the subject matter or materials discussed in the manuscript apart from those disclosed.

No writing assistance was utilized in the production of this manuscript.

5 Lewis SE, Kumar K. The paternal genome and the health of the assisted reproductive technology child. Asian. J. Androl. 17(4), 616-622 (2015).

6 Lazaraviciute G, Kauser M, Bhattacharya S, Haggarty P, Bhattacharya S. A systematic review and meta-analysis of DNA methylation levels and imprinting disorders in children conceived by IVF/ICSI compared with children conceived spontaneously. Hum. Reprod. Update 20(6), 840-852 (2014).

7 Marianowski P, Dabrowski FA, Zygula A, Wielgos M, Szymusik I. Do we pay enough attention to culture conditions in context of perinatal outcome after in vitro fertilization? Up-to-date literature review. Biomed. Res. Int. 2016, 3285179 (2016).

8 Bouillon C, Leandri R, Desch L et al. Does embryo culture medium influence the health and development of children born after in vitro fertilization? PLoS ONE 11(3), e0150857 (2016). 
9 Reik W, Dean W, Walter J. Epigenetic reprogramming in mammalian development. Science 293(5532), 1089-1093 (2001).

10 Choux C, Carmignac V, Bruno C, Sagot P, Vaiman D, Fauque P. The placenta: phenotypic and epigenetic modifications induced by assisted reproductive technologies throughout pregnancy. Clin. Epigenetics 7(1), 87 (2015).

11 Palermo GD, Neri QV, Rosenwaks Z. To ICSI or not to ICSI. Semin. Reprod. Med. 33(2), 92-102 (2015).

12 Katalinic A, Rosch C, Ludwig M, German IF-USG. Pregnancy course and outcome after intracytoplasmic sperm injection: a controlled, prospective cohort study. Fertil. Steril. 81(6), 1604-1616 (2004).

13 Sutcliffe AG, Manning JT, Katalanic A et al. Perturbations in finger length and digit ratio (2D:4D) in ICSI children. Reprod. Biomed. Online 20(1), 138-143 (2010).

14 Ceelen M, Van Weissenbruch MM, Vermeiden JP, Van Leeuwen FE, Delemarre-Van De Waal HA. Pubertal development in children and adolescents born after IVF and spontaneous conception. Hum. Reprod. 23(12), 2791-2798 (2008).

15 Belva F, Roelants M, Painter R, Bonduelle M, Devroey P, De Schepper J. Pubertal development in ICSI children. Hum. Reprod. 27(4), 1156-1161 (2012).

16 Vermeiden JP, Bernardus RE. Are imprinting disorders more prevalent after human in vitro fertilization or intracytoplasmic sperm injection? Fertil. Steril. 99(3), 642-651 (2013).

17 Loke YJ, Galati JC, Saffery R, Craig JM. Association of in vitro fertilization with global and IGF2/H19 methylation variation in newborn twins. J. Dev. Orig. Health Dis. 6(2), 115-124 (2015).

18 Wu EX, Stanar P, Ma S. X-chromosome inactivation in female newborns conceived by assisted reproductive technologies. Fertil. Steril. 101(6), 1718-1723 (2014).
19 Kohda T, Ishino F. Embryo manipulation via assisted reproductive technology and epigenetic asymmetry in mammalian early development. Philos. Trans. R. Soc. Lond. B Biol. Sci. 368(1609), 20120353 (2013).

20 Boissonnas CC, Jouannet P, Jammes H. Epigenetic disorders and male subfertility. Fertil. Steril. 99(3), 624-631 (2013).

21 Rajender S, Avery K, Agarwal A. Epigenetics, spermatogenesis and male infertility. Mutat. Res. 727(3), 62-71 (2011).

22 Van Montfoort AP, Hanssen LL, De Sutter P, Viville S, Geraedts JP, De Boer P. Assisted reproduction treatment and epigenetic inheritance. Hum. Reprod. Update 18(2), 171-197 (2012)

23 Houshdaran S, Cortessis VK, Siegmund K, Yang A, Laird PW, Sokol RZ. Widespread epigenetic abnormalities suggest a broad DNA methylation erasure defect in abnormal human sperm. PLoS ONE 2(12), e1289 (2007).

24 Yu B, Zhou H, Liu M et al. Epigenetic alterations in density selected human spermatozoa for assisted reproduction. PLoS ONE 10(12), e0145585 (2015).

25 Sharma R, Agarwal A, Rohra VK, Assidi M, Abu-Elmagd M, Turki RF. Effects of increased paternal age on sperm quality, reproductive outcome and associated epigenetic risks to offspring. Reprod. Biol. Endocrinol. 13, 35 (2015).

26 Kagami M, Nagai T, Fukami M, Yamazawa K, Ogata T. Silver-Russell syndrome in a girl born after in vitro fertilization: partial hypermethylation at the differentially methylated region of PEG1/MEST. J. Assist. Reprod. Genet. 24(4), 131-136 (2007).

27 Kobayashi H, Hiura H, John RM et al. DNA methylation errors at imprinted loci after assisted conception originate in the parental sperm. Eur. J. Hum. Genet. 17(12), 1582-1591 (2009). 